This PDF is a selection from an out-of-print volume from the National Bureau of Economic Research

Volume Title: Economics of the Family: Marriage, Children, and Human Capital

Volume Author/Editor: Theodore W. Schultz, ed.

Volume Publisher: University of Chicago Press

Volume ISBN: 0-226-74085-4

Volume URL: http://www.nber.org/books/schu74-1

Publication Date: 1974

Chapter Title: Interaction between Quantity and Quality of Children

Chapter Author: Gary S. Becker, H. Gregg Lewis

Chapter URL: http://www.nber.org/chapters/c2963

Chapter pages in book: (p. $81-90)$ 


\section{Interaction between Quantity and Quality of Children}

\section{Gary S. Becker and H. Gregg Lewis}

University of Chicago

Students of human fertility have been aware for a long time that there may be some special relation between the number (quantity) of children ever born to a family and the "quality" of their children as perceived by others if not by the parents. One need only cite the negative correlation between quantity and quality of children per family so often observed in both cross-section and time-series data. One of us (Becker 1960) more than a decade ago stressed the importance for understanding fertility (quantity) of the interaction between quantity and quality, and we are pleased to note that this interaction is emphasized in this book, and especially by De Tray and Willis.

Some economists have argued that the negative relation between quantity and quality often observed is a consequence of a low substitution elasticity in a family's utility function between parents' consumption or level of living and that of their children (see, e.g., Duesenberry 1960; Willis 1969). The approach followed by De Tray in this volume is different, but it makes equally special assumptions about the substitution between quantity and quality in the utility function and in household production.

We want to argue here that one can go a long way toward understanding data on the interaction between quantity and quality as well as on quantity or quality alone without assuming that, either in the utility function or in household production, quantity and quality are more closely related than any two commodities chosen at random. The analysis that follows is sketchy and incomplete, mainly because we have only recently developed this line of argument.

The key feature in our analysis is that the shadow price of children with respect to their number (i.e., the cost of an additional child, holding their quality constant) is greater the higher their quality is. Similarly, the shadow price of children with respect to their quality (i.e., the cost 
of a unit increase in quality, holding number constant) is greater, the greater the number of children. Furthermore, with appropriate change of language, the same may be said of the other commodities consumed by the family. However, to simplify the analysis in this paper, we make the quantity-quality distinction only for children. Thus, to illustrate our reasoning, we specify the following simple utility function:

$$
U=U(n, q, y) \text {, }
$$

where $n$ is the number of children, $q$ their quality (assumed to be the same for all of the children), and $y$ the rate of consumption of all other commodities. We start out with a simple budget restraint:

$$
I=n q \pi+y \pi_{y},
$$

where $I$ is full income, $\pi$ is the price of $n q$, and $\pi_{y}$ is the price of $y$. We make no special assumptions about the elasticities of substitution among $n, q$, and $y$, either in the utility function or in the household production functions that underlie the $\pi$ 's.

The first-order conditions for maximizing the utility function subject to the budget restraint are:

$$
M U_{n}=\lambda q \pi=\lambda p_{n} ; M U_{q}=\lambda n \pi=\lambda p_{q} ; M U_{y}=\lambda \pi_{y}=\lambda p_{y},
$$

where the $M U$ 's are the marginal utilities, the $p$ 's are marginal costs or shadow prices, and $\lambda$ is the marginal utility of money income. The important point is that the shadow price of children with respect to number $\left(p_{n}\right)$ is positively related to $q$, the level of quality, and the shadow price with respect to quality $\left(p_{q}\right)$ is positively related to $n$, the number of children. The economic interpretation is that an increase in quality is more expensive if there are more children because the increase has to apply to more units; similarly, an increase in quantity is more expensive if the children are of higher quality, because higher-quality children cost more.

These equilibrium conditions ( 3 ) together with the second-order conditions can be found in several places in the literature on quantity and quality (see, e.g., Houthakker 1952; Theil 1952; Becker 1960; and Willis's paper in this volume), but a number of their important implications for income and price effects apparently have not been explored.

\section{Income Effects}

Let the "true" income elasticities of demand for the number $(n)$ and quality $(q)$ of children and for all other commodities $(y)$ be $\eta_{n}, \eta_{q}$, and $\eta_{\nu}$, respectively. These elasticities are derived in the usual way by changing "income" while holding constant the "prices" of $n, q$, and $y$. The appropriate prices for this purpose are the shadow prices (marginal costs) 
$p_{n}, p_{q}$, and $p_{y}$, whose ratios in equilibrium (see eq. [3]) are equal to the marginal rates of substitution in the utility function. The appropriate income concept is the total "expenditure" on $n, q$, and $y$ calculated at these shadow prices; that is, the correct measure of income for this purpose is

$$
R=n p_{n}+q p_{q}+y p_{y}=I+n q \pi .
$$

It is well known that the mean value of the true income elasticities is unity; that is:

$$
1=\frac{n p_{n}}{R} \eta_{n}+\frac{q p_{q}}{R} \eta_{q}+\frac{y p_{\nu}}{R} \eta_{\nu} .
$$

Consider, however, the "observed" income elasticities, which we denote by $\bar{\eta}_{n}, \bar{\eta}_{q}$, and $\bar{\eta}_{y}$, derived by changing $I$ while holding $\pi$ and $\pi_{y}$ constant. It follows directly from the budget restraint (2) and the definitions of the $p$ 's in (3) that the similarly weighted mean of the observed income elasticities is $I / R=I /(I+n q \pi)$, which is less than unity; that is:

$$
1>\frac{I}{R}=\frac{I}{I+n q \pi}=\frac{n p_{n}}{R} \bar{\eta}_{n}+\frac{q p_{Q}}{R} \bar{\eta}_{q}+\frac{y p_{v}}{R} \bar{\eta}_{\nu} .
$$

That is, on the average, the observed elasticities are smaller than the true elasticities in the ratio $I / R$. The economic explanation for this downward bias is simple. The direct effect of the increase in $I$, holding the $\pi$ 's but not the p's constant, in general is to increase $n, q$, and $y$. However, increases in $n$ and $q$ cause the shadow prices $p_{n}$ and $p_{\eta}$ to rise. Thus, the percentage increase in real income in the sense of $R$ deflated by an index of the $p$ 's is less than the percentage increase in money income $I$.

This price effect of an increase in money income resembles somewhat the price effect resulting from a rise in money income caused by a rise in wage rates. The increase, in ratio terms, is less in real income than in money income, because the costs of producing commodities in the household are increased by the rise in the price of time (see Becker 1965). ${ }^{1}$

We think that it is plausible to assume that the true income elasticity with respect to quality $\left(\eta_{q}\right)$ is substantially larger than that with respect to quantity $\left(\eta_{n}\right)$. Because of the downward bias in the observed elasticities and the effect on prices, the observed elasticity for quantity $\left(\bar{\eta}_{n}\right)$ may be negative even though the true elasticity $\left(\eta_{n}\right)$ is not. Assume for simplicity

1 This price effect, however, does offer a correction to the argument advanced by Becker (1960), and followed by many others, that the price of children is the same for the rich as for the poor (aside from the cost-of-time argument), even though the rich choose more expensive children. The relevant price of children with respect to their number is higher for the rich precisely because they choose more expensive children. Similarly; the relevant price of cars, houses, or other goods is higher for the rich because they choose more expensive varieties. 
that $\eta_{n}=0$. Let income $I$ increase while holding $\pi$ and $\pi_{y}$ constant. The direct effect of the increase in $I$ is to increase $q$ (and $y$ ) while leaving $n$ unchanged. But then the shadow price with respect to quantity $\left(p_{n}=q \pi\right)$ will rise while the shadow price of quality $\left(p_{q}=n \pi\right)$ and that of $y\left(p_{y}=\right.$ $\pi_{y}$ ) are unchanged, causing $q$ and $y$ to be substituted for $n$, and therefore $n$ will decline.

More generally, when the utility function and budget restraints are those given above in equations (1) and (2), the observed income elasticities for quantity and quality are related to the corresponding true elasticities as follows: ${ }^{2}$

$$
\begin{aligned}
& \frac{D \bar{\eta}_{n}}{1-k}=\left(1-k \sigma_{n q}\right) \eta_{n}-(1-k) \bar{\sigma}_{n} \eta_{q} ; \\
& \frac{D \bar{\eta}_{q}}{1-k}=\left(1-k \sigma_{n q}\right) \eta_{q}-(1-k) \bar{\sigma}_{q} \eta_{n},
\end{aligned}
$$

where

$$
\left\{\begin{aligned}
k & \equiv \frac{n q \pi}{R} ;(1-k) \bar{\sigma}_{n}=k \sigma_{n q}+(1-2 k) \sigma_{n y} ;(1-k) \bar{\sigma}_{q} \\
& =k \sigma_{n q}+(1-2 k) \sigma_{q \nu} ; \\
D & \equiv\left(1-k \sigma_{n q}\right)^{2}-(1-k)^{2} \bar{\sigma}_{n} \bar{\sigma}_{q} .
\end{aligned}\right.
$$

The $\sigma$ 's are the familiar Allen partial elasticities of substitution in the utility function; the $\bar{\sigma}$ 's are averages of the $\sigma$ 's, and they must be positive; $D$ and $\left(1-k \sigma_{n q}\right)$ are positive by the second-order conditions. Equations (7) verify that the observed quantity elasticity $\left(\bar{\eta}_{n}\right)$ may be negative even when the true quantity elasticity $\left(\eta_{n}\right)$ is positive. Furthermore, if $\eta_{q}>\eta_{n}$, as we assume, $\bar{\eta}_{q}>\bar{\eta}_{n}$ unless $q$ is a much better substitute than $n$ for $y$, for it follows from (7) and (8) that

$$
\frac{D}{1-k}\left(\bar{\eta}_{q}-\bar{\eta}_{n}\right)=\eta_{q}-\eta_{n}+(1-2 k)\left(\sigma_{n \nu} \eta_{q}-\sigma_{q y} \eta_{n}\right)
$$

Moreover, $\bar{\eta}_{q}$ may exceed $\bar{\eta}_{n}$ by more than $\eta_{q}$ exceeds $\eta_{n}$; that is, the downward bias in $\bar{\eta}_{q}$ may be less than that in $\bar{\eta}_{n}$. This is easily seen for the case in which $\sigma_{n q}=\sigma_{n y}=\sigma_{q \nu}=\sigma$. Then $D$, which is positive, is equal to $(1-\sigma)[1+\sigma(1-2 k)]$ and $\bar{\eta}_{q}-\bar{\eta}_{n}=(1-k)\left(\eta_{q}-\eta_{n}\right) /$ $(1-\sigma)$, so that $\bar{\eta}_{q}-\bar{\eta}_{n}>\eta_{q}-\eta_{n}$ if $\sigma>k$. Indeed, $\bar{\eta}_{q}$ may even exceed $\eta_{q}$, as may be seen from (7) and (8) by assuming $\eta_{n}=0$ and $\sigma_{n q}=\sigma_{n \nu}=\sigma_{q \nu}=\sigma$. Then $\bar{\eta}_{q}=(1-k)(1-k \sigma) \eta_{q} /(1-\sigma)[1+$ $\sigma(1-2 k)]$, which will exceed $\eta_{Q}$ if, for example, $k=1 / 3$ and $\sigma=3 / 4$.

2 See the Mathematical Appendix to this paper. 
Even if $\eta_{n}$ were constant, $\bar{\eta}_{n}$ need not be, since the latter depends not only on $\eta_{n}$ but also on the substitution elasticities and the share of $n q \pi$ in money income $I(n q \pi / I=k /(1-k))$. For example, if $\eta_{q}$ declines as income $I$ rises-a plausible assumption, we think- $\bar{\eta}_{n}$ would tend to rise with income, even with constant $\eta_{n}$, and, of course, $\eta_{n}$ may rise with income, contributing to the increase in $\bar{\eta}_{n}$. Indeed, $\bar{\eta}_{n}$ could be negative at lower levels of income and positive at higher levels, the pattern observed in some fertility data. ${ }^{3}$

\section{Price Effects}

Before discussing price effects, we generalize the budget constraint (2) slightly as follows:

$$
I=n \pi_{n}+n q \pi+q \pi_{q}+y p_{\nu}
$$

so that the shadow prices or marginal costs are now

$$
p_{n}=\pi_{n}+q \pi ; p_{q}=\pi_{q}+n \pi ; p_{y}=\pi_{y} .
$$

These shadow prices for $n$ and $q$ each contain a "fixed" component: $\pi_{n}$ in $p_{n}$ and $\pi_{q}$ in $p_{q}$. The component $n \pi_{n}$ in child costs consists of costs that depend on quantity but not on quality. Contraception costs and prenatal child costs (such as maternity care) are moderately good examples. Similarly, the component $q \pi_{q}$ depends on quality but not quantity, and thus has the attributes of a "public good," or a better expression is a "family good." Perhaps some aspects of training in the home and the "handing down" of some clothing are reasonable examples. We assume that the fixed component is more important for quantity than for quality, that is, $n \pi_{n}>q \pi_{q}$.

a) First consider the pure substitution effects of an increase in $\pi_{n}$ induced, say, by an exogenous improvement in contraceptive technique. Since this increases the shadow price of quantity $\left(p_{n}\right)$ relative to both the shadow price of quality $\left(p_{q}\right)$ and the shadow price of $y\left(p_{y}\right), n$ would fall. But the fall in numbers reduces the shadow price of quality $\left(p_{q}=\pi_{q}+\right.$ $n \pi$ ), which induces substitution in favor of quality. The outcome would be not only a fall in quantity but also a relatively large rise in qualityrelative, that is, to other commodities-without assuming that quantity and quality are better substitutes than any two commodities chosen at random. Exactly the same result holds if $\pi_{q}$ falls, say, because of an increase in the education of parents. The fall in $p_{q}$ induces an increase in quality, which in turn induces an increase in the shadow price of quantity $\left(p_{n}=\pi_{n}+q \pi\right)$ and thus a relatively large decrease in quantity.

Consequently, both De Trav's finding (in his paper which follows) that an

\footnotetext{
${ }^{3}$ See the discussion and alternative explanation of this finding earlier in Willis's paper.
} 
increase in the education of mothers has a strong positive effect on the quality and a strong negative effect on the number of their children, and the common belief that important advances in birth control knowledge not only significantly reduce the number of children but also significantly increase their quality, are consistent with the preceding analysis. Quantity and quality are closely related, because the shadow price of quality depends on quantity and the shadow price of quantity depends on quality. We repeat that no special assumptions about substitution in household production or consumption are required to derive a special relation between quantity and quality.

b) Now consider the pure substitution effects of equal percentage increases in $\pi_{n}, \pi_{q}$, and $\pi$ due, say, to increases in wage rates. To put the argument in extreme form, assume $\pi_{q}=0$ and $\pi_{n}>0$. The equal increases in $\pi_{n}, \pi_{q}$, and $\pi$ relative to $\pi_{y}=p_{y}$ can be treated simply as a relative fall in $\pi_{y}=p_{y}$. A fall in $p_{y}$ initially would induce equal percentage declines in $n$ and $q$ if they were equally good substitutes for $y$. However, since the equal percentage declines in $n$ and $q$ would lower $p_{q}$ more than $p_{n}, n$ would fall relative to $q$. Thus, the income-compensated elasticity of quantity with respect to equal percentage changes in $\pi_{n}, \pi_{q}$ and $\pi$ tends to be greater numerically than the corresponding elasticity for quality. De Tray finds that an increase in women's wage rates reduces the number of children by a much bigger percentage than the quality of children.

This difference is, of course, accentuated if quantity is a better substitute than quality for other commodities, which we think is a plausible, though special, assumption. For then a fall in $p_{y}$ directly induces a fall in $n$ relative to $q$, which accentuates the decline in $p_{q}$ relative to $p_{n}$.

We conclude, therefore, that the observed price elasticity of quantity exceeds that of quality, just the opposite of our conclusion for observed income elasticities. ${ }^{4}$ This reversal of the quantity-quality ordering for price and income elasticities is not only a somewhat unexpected implication of the analysis, but also gives a consistent interpretation to the findings of De Tray and others.

Of course, most of our discussion applies not only to the interaction between the quantity and quality of children, but also to the quantity and quality of cars, houses, food, tea, education, publications, and large numbers of other goods. The observed price and income elasticities of quantity and quality will differ in predictable directions from the "true" elasticities. A systematic analysis and reconsideration of the interaction between quan-

4 This conclusion about income elasticities, derived from the budget restraint (2), is modified somewhat when the budget restraint is of the more general form (10), since the shadow price of quality is less sensitive to any given percentage change in quality than the price of quality is to a change in quantity. Conceivably then, $\bar{\eta}_{n}$ could be greater than $\bar{\eta}_{q}$ at the same time that $\eta_{n}<\eta_{q}$, but we consider this unlikely, since $\eta_{q}$ is probably much greater than $\eta_{n}$. 
tity and quality of all goods from the viewpoint of this paper should be quite rewarding.

\section{Mathematical Appendix}

The budget restraints specified in equations $(2)$ and $(10)$ are not linear in $n$ and $q$. It is precisely this nonlinearity, of course, that leads to the "interaction between quantity and quality" that we discuss in this paper.

The derivation of the elasticities of the demand functions for number of children $(n)$ and child quality $(q)$ can be carried in a direct fashion by differentiating the budget restraint and the first-order conditions. Because of the nonlinearity of the budget restraint, however, if this direct mode of derivation is followed, it is all too easy to lose sight of the underlying income and substitution elasticities in the utility function. Hence, we follow an indirect approach that makes use of quite familiar propositions in demand theory.

First, we replace the curvilinear budget surface given in equation (10) by a plane surface by adding $n q \pi$ to both sides:

$$
\begin{gathered}
I+n q \pi=n\left(\pi_{n}+q \pi\right)+q\left(\pi_{q}+n \pi\right)+y \pi_{y} \\
R=n p_{n}+q p_{q}+y p_{y} .
\end{gathered}
$$

where

$$
R=I+n q \pi=I /(1-k) ; k \equiv n q \pi / R .
$$

The two income concepts $I$ and $R$ differ by the nonlinear term $n q \pi$ in the budget restraint.

Define

$$
k_{i} \equiv \frac{i p_{i}}{R}, i=n, q, y ; k_{n}+k_{q}+k_{y}=1
$$

It is well known that the true income elasticities $\left(\eta_{n}, \eta_{q}\right.$, and $\left.\eta_{y}\right)$ must satisfy the relation

$$
1=k_{n} \eta_{n}+k_{q} \eta_{q}+k_{y} \eta_{y}
$$

The observed income elasticities $\left(\bar{\eta}_{n}, \bar{\eta}_{q}, \bar{\eta}_{y}\right)$ obtained by changing full income $I$ while holding the $\pi$ 's constant, however, must satisfy

$$
1-k=-\frac{I}{R}=k_{n} \bar{\eta}_{n}+k_{q} \bar{\eta}_{q}+k_{y} \bar{\eta}_{y} \text {. }
$$

Equation (A6) may be verified by differentiating the budget restraint (10) logarithmically with respect to $I$, holding the $\pi$ 's constant, and then using equations (A3) and (A4). Thus, the observed elasticities, on the average, are smaller than the true elasticities in the ratio $1-k=1 / R$.

We now define two household price indexes $\bar{\pi}$ and $\bar{p}$ in differential form as follows:

$$
\left\{\begin{aligned}
E \bar{\pi} & \equiv \frac{y \pi_{y}}{I} E \pi_{y}+\frac{n \pi_{n}}{I} E \pi_{n}+\frac{q \pi_{q}}{I} E \pi_{g}+\frac{n q \pi}{I} E \pi \\
& =\frac{1}{1-k}\left[k_{y} E \pi_{y}+\left(k_{n}-k\right) E \pi_{n}+\left(k_{q}-k\right) E \pi_{q}+k E \pi\right],
\end{aligned}\right.
$$




$$
E \bar{p} \equiv k_{y} E p_{y}+k_{n} E p_{n}+k_{q} E p_{q}
$$

where the symbol $E$ denotes the natural logarithmic differential operator $d l n$.

Since $p_{y}=\pi_{y}, p_{n}=\pi_{n}+q \pi$, and $p_{q}=\pi_{q}+\eta_{\pi}$, it follows that

$$
\begin{aligned}
& E p_{\nu}=E \pi_{y} ; E p_{n}=\frac{\left(k_{n}-k\right) E \pi_{n}+k(E \pi+E q)}{k_{n}} ; \\
& E p_{q}=\frac{\left(k_{q}-k\right) E \pi_{q}+k(E \pi+E n)}{k_{q}} .
\end{aligned}
$$

Substitute these results (A9) into (A8) and then use (A7) to obtain

$$
E \bar{p}=(1-k) E \bar{\pi}+k E n q \pi \text {. }
$$

Now differentiate (A3) logarithmically:

$$
E R=(1-k) E I+k E n q \pi .
$$

Subtract (A10) from (A11):

$$
E(R / \bar{p})=(1-k) E(I / \bar{\pi}) .
$$

When $l$ is increased, holding the $\pi$ 's constant, real income $R / \bar{D}$ increases in the smaller ratio $1-k=I / R$. This is the economic basis of the downward bias in the observed income elasticities relative to the true income elasticities.

We now turn to the derivation of the observed income and substitution elasticities. We make use of the well-known propositions that

$$
\left\{\begin{array}{c}
E n=\eta_{n} E(R / \bar{p})+k_{y} \sigma_{n y} E p_{y}-\left(1-k_{n}\right) \bar{\sigma}_{n} E p_{n}+k_{q} \sigma_{n q} E p_{q} \\
E q=\eta_{q} E(R / \bar{p})+k_{y} \sigma_{q \nu} E p_{y}+k_{n} \sigma_{n q} E p_{n}-\left(1-k_{q}\right) \bar{\sigma}_{q} E p_{q} \\
\left(1-k_{n}\right) \bar{\sigma}_{n} \equiv k_{y} \sigma_{n y}+k_{q} \sigma_{n q} ;\left(1-k_{q}\right) \bar{\sigma}_{q}=k_{\nu} \sigma_{q y}+k_{n} \sigma_{n q}
\end{array}\right.
$$

where the $\sigma$ 's are the Allen partial elasticities of substitution in the utility function. Notice that $\bar{\sigma}_{n}$ is the average elasticity of substitution of $n$ against $y$ and $q$ and that $\bar{\sigma}_{q}$ is the similar elasticity for $q$ against $y$ and $n$.

We first derive the observed income elasticities $\bar{\eta}_{n}$ and $\bar{\eta}_{q}$ by letting $I$ change while the $\pi$ 's are constant. Because the $\pi$ 's are constant, it follows from (A9) and (A12) that

$$
E p_{y}=0 ; E p_{n}=\frac{k E q}{k_{n}}, E p_{q}=\frac{k E n}{k_{q}} ; E(R / \bar{p})=(1-k) E I .
$$

Substitute (A14) into (A13) and collect terms to obtain

$$
\left\{\begin{array}{l}
\left(1-k \sigma_{n q}\right) \bar{\eta}_{n}+\frac{k\left(1-k_{n}\right) \bar{\sigma}_{n}}{k_{n}} \bar{\eta}_{q}=(1-k) \eta_{n} \\
\frac{k\left(1-k_{q}\right) \bar{\sigma}_{q}}{k_{q}} \bar{\eta}_{n}+\left(1-k \sigma_{n q}\right) \bar{\eta}_{q}=(1-k) \eta_{q} .
\end{array}\right.
$$

Solve these two equations for $\bar{\eta}_{n}$ and $\bar{\eta}_{\boldsymbol{q}}$ : 


$$
\left\{\begin{array}{l}
\frac{D \tilde{\eta}_{n}}{1-k}=\left(1-k \sigma_{n q}\right) \eta_{n}-\frac{k\left(1-k_{n}\right) \bar{\sigma}_{n}}{k_{n}} \eta_{q}, \\
\frac{D \bar{\eta}_{q}}{1-k}=\left(1-k \sigma_{n q}\right) \eta_{q}-\frac{k\left(1-k_{q}\right) \bar{\sigma}_{q}}{k_{q}} \eta_{n}, \\
D \equiv\left(1-k \dot{\sigma}_{n q}\right)^{2}-\frac{k^{2}\left(1-k_{n}\right)\left(1-k_{q}\right) \bar{\sigma}_{n} \bar{\sigma}_{q}}{k_{n} k_{q}},
\end{array}\right.
$$

where $D$ and $\left(1-k \sigma_{n q}\right)$ must be positive by the second-order conditions.

In the section on income effects, we used the simpler budget restraint (2) rather than (10), so that we assumed that $k_{n}=k_{q}=k$; equations (7) and (8) are simply equations (A16) when $k_{n}=k_{q}=k$. The only proposition in that section that needs qualification when the budget restraint is $(10)$ is the proposition that $\bar{\eta}_{q}-\bar{\eta}_{n}$ has the sign of $\eta_{q}-\eta_{n}$ unless $\sigma_{q \nu}$ is much larger than $\sigma_{n y}$. Let $\sigma_{n y}=\sigma_{q \nu}=\sigma_{n q}=\sigma$ in (A16). Then

$$
\frac{D\left(\bar{\eta}_{q}-\bar{\eta}_{n}\right)}{1-k}=(1-2 k \sigma)\left(\eta_{q}-\eta_{n}\right)+\frac{k \sigma}{k_{n} k_{q}}\left(k_{q} \eta_{q}-k_{n} \eta_{n}\right),
$$

where $(1-2 k \sigma)$ must be positive by the second-order conditions. In the section on price effects, we assume that $k_{n}-k_{q}=n \pi_{n}-q \pi_{q} / R$ is positive. But then if $\eta_{q} / \eta_{n}$ is sufficiently smaller than $k_{n} / k_{q}, \bar{\eta}_{q}-\bar{\eta}_{n}$ will have a sign opposite that of $\eta_{q}-\eta_{n}$. We have noted this qualification and commented on it (see n. 4 above).

We turn now to the income-compensated elasticities of quantity and quality with respect to the $\pi$ 's, deriving them in essentially the same manner as the income elasticities. We consider first the elasticities with respect to $\pi_{n}$ and $\pi_{q}$ :

$$
\left\{\begin{array}{l}
D \bar{\eta}_{i \pi_{i}}{ }^{(s)}=-\frac{\left(k_{i}-k\right)\left(1-k_{i}\right) \bar{\sigma}_{i}}{k_{i}}, i=n, q, \\
D \bar{\eta}_{i \pi_{j}}{ }^{(s)}=\left(k_{j}-k\right)\left[\sigma_{n q}\left(1-k \sigma_{n q}\right)+\frac{k\left(1-k_{i}\right)\left(1-k_{j}\right) \bar{\sigma}_{i} \bar{\sigma}_{j}}{k_{i} k_{j}}\right], \\
i \neq j=n, q . \\
\text { (A18) }
\end{array}\right.
$$

When $\sigma_{n y}=\sigma_{q y}=\sigma_{n q}=\sigma$, these simplify to

$$
\left\{\begin{aligned}
D \bar{\eta}_{i \pi_{i}}{ }^{(s)} & =-\frac{\left(k_{i}-k\right)\left(1-k_{i}\right) \sigma}{k_{i}}, i=n, q, \\
D \bar{\eta}_{i \pi_{j}}{ }^{(s)} & =\frac{\left(k_{j}-k\right) \sigma}{k_{i} k_{j}}\left(k_{i} k_{j}+k k_{y} \sigma\right), i \neq j=n, q, \\
\mathrm{D} & =(1-2 k \sigma)-\frac{k_{y}(k \sigma)^{2}}{k_{n} k_{q}} .
\end{aligned}\right.
$$

Both observed "own price" elasticities, $\bar{\eta}_{n \pi_{n}}{ }^{(8)}$ and $\bar{\eta}_{q \pi_{q}}{ }^{(8)}$ are negative, and the sign of $\left|\bar{\eta}_{n \pi_{n}}{ }^{(s)}\right|-\left|\bar{\eta}_{q \pi_{q}}{ }^{(\theta)}\right|$ is ambiguous even when it is assumed that 
$\sigma_{n y}=\sigma_{q y}=\sigma_{n q}=\sigma$ and that $k_{n}-k_{q}>0$. However, if $\pi_{q}$ is small relative to $p_{q},\left|\bar{\eta}_{n \pi_{n}}{ }^{(s)}\right|$ will exceed $\left|\bar{\eta}_{q \pi_{q}}{ }^{(s)}\right|$. The observed cross-elasticities, $\bar{\eta}_{n \pi_{q}}{ }^{(s)}$ and $\bar{\eta}_{q \pi_{n}}{ }^{(8)}$, are positive if $\sigma_{n q}>0$, and $\bar{\eta}_{q \pi_{n}}{ }^{(8)}$ exceeds $\bar{\eta}_{n \pi_{q}}{ }^{(8)}$ if $k_{n}>k_{q}$, as we assume. The cross-partial derivatives, however, are equal:

$$
\frac{\partial n}{\partial \pi_{q}}=\frac{\partial q}{\partial \pi_{n}}=\frac{n q}{R}\left[\sigma_{n q}\left(1-k \sigma_{n q}\right)+\frac{k\left(1-k_{n}\right)\left(1-k_{q}\right) \bar{\sigma}_{n} \bar{\sigma}_{q}}{k_{n} k_{q}}\right]
$$

where the derivatives are income-compensated.

The observed elasticities with respect to $\pi_{y}=p_{y}$ are

$$
\frac{D \bar{\eta}_{i \pi_{y}}}{k_{y}}=\sigma_{i v}\left(1-k \sigma_{n q}\right)-\sigma_{j y} k\left(k_{j} \sigma_{n q}+k_{\nu} \sigma_{i v}\right) / k_{i}, i \neq j=n, q,
$$

from which it follows that

$$
\left\{\begin{aligned}
\frac{D}{k_{y}}\left(\bar{\eta}_{n \pi_{y}}{ }^{(s)}-\bar{\eta}_{q \pi_{y}}{ }^{(s)}\right) & =\left(\sigma_{n \nu}-\sigma_{q \nu}\right)\left(1-k \sigma_{n q}\right) \\
+ & \frac{k \sigma_{n q}}{k_{n} k_{q}}\left(k_{n}^{2} \sigma_{n y}-k_{q}^{2} \sigma_{q \nu}\right)+\frac{k k_{y} \sigma_{n y} \sigma_{q \nu}}{k_{n} k_{q}}\left(k_{n}-k_{q}\right) .
\end{aligned}\right.
$$

Thus, if the $\sigma$ 's are equal and if $k_{n}>k_{q}$, the quantity elasticity $\left(\bar{\eta}_{n \pi_{y}}{ }^{(8)}\right.$ ) exceeds the quality elasticity $\left(\bar{\eta}_{q \pi_{y}}{ }^{(g)}\right)$; this difference is increased if $\sigma_{n y}>\sigma_{q y}$, a special, though plausible, assumption.

The observed elasticities with respect to equal percentage changes in $\pi_{n}, \pi_{q}$, and $\pi$ are simply those with respect to $\pi_{y}$ but with signs changed. Thus, the demand functions for $n$ and $q$ are homogeneous of degree zero in $I$ and the $\pi$ 's, just as they are in the shadow income $(R)$ and the shadow prices ( $p$ 's). 